\title{
On the cohomology of Fano varieties and the Springer correspondence
}

\section{Chen, Tsao-Hsien}

2017-10-01

Chen , T-H , Vilonen , K , Xue , T \& Stanton , D 2017 , ' On the cohomology of Fano varieties and the Springer correspondence ' , Advances in Mathematics , vol. 318 , pp. 515-533 . https://doi.org/10.1016/j.aim

http://hdl.handle.net/10138/307131

https://doi.org/10.1016/j.aim.2017.08.008

cc_by_nc_nd

acceptedVersion

Downloaded from Helda, University of Helsinki institutional repository.

This is an electronic reprint of the original article.

This reprint may differ from the original in pagination and typographic detail.

Please cite the original version. 


\title{
ON THE COHOMOLOGY OF FANO VARIETIES AND THE SPRINGER CORRESPONDENCE
}

\author{
TSAO-HSIEN CHEN, KARI VILONEN, AND TING XUE, \\ WITH AN APPENDIX BY DENNIS STANTON
}

\begin{abstract}
In this paper we compute the cohomology of the Fano varieties of $k$-planes in the smooth complete intersection of two quadrics in $\mathbb{P}^{2 g+1}$, using Springer theory for symmetric spaces.
\end{abstract}

\section{INTRODUCTION}

In this paper we compute the cohomology of the Fano varieties $\mathrm{Fano}_{k}$ of $k$-planes in the smooth complete intersection of two quadrics in $\mathbb{P}^{2 g+1}$, with $g \geq 1$. These Fano varieties have a concrete interpretation as moduli spaces of vector bundles (with extra structure) on a hyperelliptic curve $C$ of genus $g$. When $k=g-1$ then $\mathrm{Fano}_{g-1}=\operatorname{Jac}(C)$, the Jacobian of $C[\mathrm{Re}, \mathrm{Do}]$ and when $k=g-2$ then $\mathrm{Fano}_{g-2}=\operatorname{Bun}_{2}(C)$, the moduli space of stable rank 2 vector bundles on $C$ with fixed odd determinant [DR]. For $k<g-2$ a more elaborated interpretation of the varieties $\mathrm{Fano}_{k}$ as moduli spaces of bundles is given in [Ra]. The curve $C$ arises from the intersection of two quadrics in the following manner. If the intersection of the two quadrics is given by the pencil $\mu Q_{1}+\lambda Q_{2}$ then $C$ is the hyperelliptic curve over $\mathbb{P}^{1}$ ramified at the points $[\mu, \lambda]$ where the quadric $\mu Q_{1}+\lambda Q_{2}$ becomes singular.

The goal of this paper is to describe the cohomology of the varieties $\mathrm{Fano}_{k}$ in general. The form of our answer is in the spirit of the main theorem of $[\mathrm{N}]$ who, from our point of view, treats the case $\mathrm{Fano}_{g-2}$. He makes use of the mapping class group which for us, as we work with hyperelliptic curves, is replaced by the fundamental group of the universal family of hyperelliptic curves.

To state our result note that $\operatorname{dim} \mathrm{Fano}_{g-i}=(g-i+1)(2 i-1)$. We also write

$$
\bar{H}^{k}\left(\mathrm{Fano}_{g-i}, \mathbb{C}\right)=H^{\text {dim Fano }_{g-i}-k}\left(\mathrm{Fano}_{g-i}, \mathbb{C}\right), \quad \bar{\wedge}^{k}\left(H^{1}(C, \mathbb{C})\right)=\wedge^{g-k}\left(H^{1}(C, \mathbb{C})\right) .
$$

Theorem 1.1. For $i \geq 2$, we have

$$
\bar{H}^{k}\left(\operatorname{Fano}_{g-i}, \mathbb{C}\right) \cong \bigoplus_{j=i-1}^{g} N_{i}(k, j) \bar{\Lambda}^{j}\left(H^{1}(C, \mathbb{C})\right),
$$

Tsao-Hsien Chen was supported in part by the AMS-Simons travel grant.

Kari Vilonen was supported in part by NSF grants DMS-1402928 \& DMS-1069316, the Academy of Finland, the ARC grant DP150103525, the Humboldt Foundation, and the Simons Foundation.

Ting Xue was supported in part by the Academy of Finland and the ARC grant DP150103525. 
where $N_{i}(k, j)$ is the coefficient of $q^{k}$ in

$$
q^{-(j-i+1)(2 i-1)}\left(1-q^{4 j}\right) \frac{\prod_{l=j-i+2}^{i+j-2}\left(1-q^{2 l}\right)}{\prod_{l=1}^{2 i-2}\left(1-q^{2 l}\right)} .
$$

In particular, the numbers $N_{i}(k, j)$ are independent of the genus $g$.

To come up with this formula we were inspired by the case of $\mathrm{Fano}_{g-2}=\operatorname{Bun}_{2}(C)$ treated by Nelson [N]. He, in turn, states that the formula in the case of $\mathrm{FanO}_{g-2}=\operatorname{Bun}_{2}(C)$ was conjectured by Donaldson. We guessed the general formula above after reading [Z] augmented by some experimentation. By our methods we reduced the proof of the theorem to a combinatorial identity which expresses the Poincare polynomial of the orthogonal Grassmannian in terms of the Poincare polynomials of ordinary Grassmannians in a particular manner, see formula (3.13). This combinatorial identity was proved by Stanton. His proof is included in this paper as an appendix. We have not been able to understand this combinatorial identity from a geometric point of view. It would be interesting to have such a geometric interpretation.

As a byproduct one obtains formulas for the Poincare polynomials (denoted by $h_{j}^{(n)}(q)$ in the text) of stalks of IC sheaves of certain nilpotent orbits in the symmetric space case. In the classical case these stalks are given by Kostka-Foulkes polynomials. Thus, the polynomials $h_{j}^{(n)}(q)$ can be regarded as symmetric space analogues of Kostka-Foulkes polynomials. The appendix of Stanton gives an explicit formula for the $h_{j}^{(n)}(q)$ and also proves some interesting recursive formulas for them.

The proof of the theorem makes use of Springer theory for symmetric spaces which was initiated in CVX1]. In particular, it can be seen as a concrete example of a general strategy to compute the cohomology of Hessenberg varieties using the Fourier transform. In [CVX1] we primarily worked with the symmetric pair $(\mathrm{SL}(N), \mathrm{SO}(N))$ in the case when $N$ is odd. As an application of our theory we computed the cohomology of Fano varieties of $k$-planes in the complete intersection of two quadrics in an even dimensional projective space CVX1, Theorem 8.1]. In the even dimensional case the answer is considerably simpler than in the case of odd dimensional projective space we treat here. In the even dimensional case the cohomology turns out to be Hodge-Tate.

We briefly explain the idea of the proof of the theorem:

Step 1: Instead of computing the cohomology $H^{k}\left(\mathrm{Fano}_{i}, \mathbb{C}\right)$ of a single Fano variety $\mathrm{Fano}_{i}$, we put $\mathrm{Fano}_{i}$ into a proper smooth family $\pi: \widetilde{\mathrm{Fano}_{i}} \rightarrow \mathfrak{g}_{1}^{r s}$ and study the decomposition of the local system $R^{k} \pi_{*} \mathbb{C}$ into irreducibles. Here $\mathfrak{g}_{1}$ is the vector space of symmetric matrices in $\mathfrak{s l}_{2 n}(n=g+1)$ and $\mathfrak{g}_{1}^{r s}$ is the open subset consisting of regular semisimple elements.

Step 2: We consider the intersection cohomology complex $\operatorname{IC}\left(\mathfrak{g}_{1}, R^{k} \pi_{*} \mathbb{C}\right)$ associated to the local system $R^{k} \pi_{*} \mathbb{C}$. Since $R^{k} \pi_{*} \mathbb{C}$ is semisimple, the irreducible summands of $\operatorname{IC}\left(\mathfrak{g}_{1}, R^{k} \pi_{*} \mathbb{C}\right)$ are in bijection with those of $R^{k} \pi_{*} \mathbb{C}$. Hence it suffices to study the decomposition of $\mathrm{IC}\left(\mathfrak{g}_{1}, R^{k} \pi_{*} \mathbb{C}\right)$ into irreducibles. The advantage of working with $\mathrm{IC}\left(\mathfrak{g}_{1}, R^{k} \pi_{*} \mathbb{C}\right)$ is that we 
can make use of the powerful Fourier transform $\mathfrak{F}: \operatorname{Perv}\left(\mathfrak{g}_{1}, \mathbb{C}\right) \cong \operatorname{Perv}\left(\mathfrak{g}_{1}, \mathbb{C}\right)$. We show that $\mathfrak{F}\left(\mathrm{IC}\left(\mathfrak{g}_{1}, R^{k} \pi_{*} \mathbb{C}\right)\right)$ is supported on the nilpotent cone $\mathcal{N}_{1}$ in $\mathfrak{g}_{1}$ and has the form

$$
\mathfrak{F}\left(\mathrm{IC}\left(\mathfrak{g}_{1}, R^{k} \pi_{*} \mathbb{C}\right)\right) \cong \bigoplus_{j=0}^{i+1} \mathrm{IC}\left(\overline{\mathcal{O}}_{2^{j} 1^{2 n-2 j}}, \mathbb{C}^{\oplus s_{j k}}\right)
$$

where $s_{j k} \in \mathbb{Z}_{\geq 0}$ and $\mathcal{O}_{2^{j} 1^{2 n-2 j}}$ are nilpotent orbits of order two in $\mathcal{N}_{1}$ (see 92.1 ).

Step 3: We prove an explicit formula for those $s_{j k}$ (see $\$ 3.2$ ) and compute the Fourier transform of $\operatorname{IC}\left(\overline{\mathcal{O}}_{2^{i} 1^{2 n-2 i}}, \mathbb{C}\right)$. We show that the Fourier transform is given by

$$
\mathfrak{F}\left(\mathrm{IC}\left(\overline{\mathcal{O}}_{2^{i} 1^{2 n-2 i}}, \mathbb{C}\right)\right) \cong \operatorname{IC}\left(\mathfrak{g}_{1}, \mathcal{W}_{i}\right)
$$

where $\mathcal{W}_{i}$ is an irreducible local system on $\mathfrak{g}_{1}^{r s}$ coming from the universal family of hyperelliptic curves of genus $n-1$ over $\mathfrak{g}_{1}^{r s}$ (see Proposition 2.3). This can be viewed as an example of Springer correspondence for the symmetric pair $(\mathrm{SL}(2 n), \mathrm{SO}(2 n))$. Now taking the Fourier transform on both sides of (1.1) we obtain the decomposition of $\operatorname{IC}\left(\mathfrak{g}_{1}, R^{k} \pi_{*} \mathbb{C}\right)$ into irreducibles, hence that of $R^{k} \pi_{*} \mathbb{C}$. The theorem follows.

It follows from the proof that the isomorphism in Theorem 1.1 is compatible with the natural actions of the fundamental group of the universal family of hyperelliptic curves (or rather the braid group $B_{2 n}=\pi_{1}\left(\mathfrak{g}_{1}^{r s} / \mathrm{SO}(2 n)\right)$ on both sides.

As we mentioned earlier, our method of computing cohomology of Fano varieties can be applied to a more general situation, namely, the case of Hessenberg varieties. Those varieties, first introduced in [DPS], play an important role in algebraic geometry and representation theory. For example, in CVX2] we show that complete intersections of quadrics are examples of Hessenberg varieties, and in [GKM, OY, T] the authors show that many important questions in harmonic analysis on $p$-adic groups and representations of Cherednik algebras are closely related to cohomology of Hessenberg varieties. It would be very interesting to understand the cohomology of those Hessenberg varieties from the perspective of our method.

The paper is organized as follows. In Section 2 we explain how the theory developed in CVX1] for odd $N$ can be carried over to the case of even $N$ and we establish the Springer correspondence for nilpotent orbit of order two for the symmetric pair ( $\mathrm{SL}(2 n), \mathrm{SO}(2 n))$. In Section 3 we give the proof of our main theorem. The appendix by Dennis Stanton contains the proof of the combinatorial identity.

Acknowledgements. We thank the Max Planck Institute for Mathematics in Bonn for support, hospitality, and a nice research environment. Furthermore KV and TX thank the Research Institute for Mathematical Sciences in Kyoto for support, hospitality, and a nice research environment. Special thanks are due to Dennis Stanton for proving a key combinatorial identity and for writing an appendix containing the proof. 


\section{SPRINGER CORRESPONDENCE FOR the SyMmetric PAIR ( $\mathrm{SL}(2 n), \mathrm{SO}(2 n))$}

In this section we discuss the Springer correspondence for nilpotent orbits of order 2 for the symmetric pair $(\mathrm{SL}(2 n), \mathrm{SO}(2 n))$. We have treated the case $(\mathrm{SL}(2 n+1), \mathrm{SO}(2 n+1))$ in CVX1]. Some of the arguments in this section are modifications of the arguments in [CVX1. In this section we have written $n$ for $g+1$ and $n \geq 2$.

We follow the notational conventions of [CVX1]. In particular, we adopt the usual convention of cohomological degrees for perverse sheaves by having them be symmetric around 0 . We also use the convention that all functors are derived, so we write, for example, $\pi_{*}$ instead of $R \pi_{*}$. If $X$ is smooth we write $\mathbb{C}_{X}[-]$ for the constant sheaf placed in degree $-\operatorname{dim} X$ so that $\mathbb{C}_{X}[-]$ is perverse. If $U \subset X$ is a smooth open dense subset of a variety $X$ and $\mathcal{L}$ is a local system on $U$, we write $\operatorname{IC}(X, \mathcal{L})$ for the IC-extension of $\mathcal{L}[-]$ to $X$; in particular, it is perverse. For $\mathcal{F} \in \mathrm{D}(X)$ and $x \in X$, we write $\mathcal{H}_{x}^{i}(\mathcal{F})$ for the stalk of the cohomology sheaf $\mathcal{H}^{i} \mathcal{F}$ at $x$. This should not be confused with local cohomology. For a number $a$, we denote the integer part of $a$ by $[a]$.

2.1. The symmetric pair $(\operatorname{SL}(2 n), \operatorname{SO}(2 n))$. Let $G=\operatorname{SL}(2 n, \mathbb{C})$ and $\theta: G \rightarrow G$ an involution such that $K:=G^{\theta}=\mathrm{SO}(2 n, \mathbb{C})$. We also write $(G, K)=(\operatorname{SL}(V), \mathrm{SO}(V, Q))$, where $\operatorname{dim} V=2 n$. We think of $Q$ concretely as a non-degenerate quadratic form on $V$ and we write $\langle,\rangle_{Q}$ for the non-degenerate bilinear form on $V$ associated to $Q$. The involution $\theta$ induces a grading on $\mathfrak{g}=$ Lie $G$, i.e. $\mathfrak{g}=\mathfrak{g}_{0} \oplus \mathfrak{g}_{1}$, where $\left.\theta\right|_{\mathfrak{g}_{i}}=(-1)^{i}$. If we diagonalize $Q$ then the Cartan involution $\theta$ is given by $g \mapsto\left(g^{t}\right)^{-1}$ and then $\mathfrak{g}_{1}$ consists of symmetric matrices.

The pair $(G, K)$ is a split pair. We write $\mathfrak{g}^{\text {rs }}$ for the regular semi-simple elements in $\mathfrak{g}$ and we let $\mathfrak{g}_{1}^{r s}=\mathfrak{g}_{1} \cap \mathfrak{g}^{r s}$. Furthermore, we write $A$ for a $\theta$-stable maximal split torus of $G$, i.e., $\theta(t)=t^{-1}$ for $t \in A$. We write $\pi_{1}^{K}\left(\mathfrak{g}_{1}^{r s}\right)$ for the equivariant fundamental group of $\mathfrak{g}_{1}^{r s}$ and we have

$$
\pi_{1}^{K}\left(\mathfrak{g}_{1}^{r s}\right)=A[2] \rtimes B_{2 n},
$$

where $A[2]$ is the group of order 2 elements in $A$ and $B_{2 n}$ is the braid group. For a discussion of these matters see [CVX1, §2.6].

We also write $\mathcal{N}$ for the nilpotent cone of $\mathfrak{g}$ and we let $\mathcal{N}_{1}=\mathcal{N} \cap \mathfrak{g}_{1}$ stand for the nilpotent cone of $\mathfrak{g}_{1}$. The $G$-orbits in $\mathcal{N}$ are parametrized by partitions $\lambda$ of $2 n$ and we write $\tilde{\mathcal{O}}_{\lambda}$ for the $G$-orbit corresponding to the partition $\lambda$. The intersection of a $G$-orbit $\tilde{\mathcal{O}}_{\lambda}$ with $\mathcal{N}_{1}$ is one $K$-orbit in the case when not all parts of $\lambda$ are even and decomposes into two $K$-orbits otherwise [S].

For $i \in[0, n-1]$, let $\mathcal{O}_{2^{i} 1^{2 n-2 i}}$ denote the (unique) nilpotent $K$-orbit in $\mathfrak{g}_{1}$ corresponding to the partition $\lambda=2^{i} 1^{2 n-2 i}$, where $i$ (resp. $2 n-2 i$ ) is the multiplicity of 2 (resp. 1 ) in $\lambda$. Let us denote by $\operatorname{OGr}(s, 2 n)$ the variety of $s$-dimensional isotropic subspaces in $\mathbb{C}^{2 n}$ with respect to a non-degenerate bilinear form. We write $V_{i}$ for a vector subspace of $V=\mathbb{C}^{2 n}$ of dimension $i$ and $V_{i}^{\perp}=\left\{x \in V \mid\left\langle x, V_{i}\right\rangle_{Q}=0\right\}$. 
2.2. Reeder's resolutions for $\overline{\mathcal{O}}_{2^{i} 1^{2 n-2 i}}$. Consider the natural projection maps

$$
v_{i}:\left\{\left(x, 0 \subset V_{i} \subset V_{i}^{\perp} \subset \mathbb{C}^{2 n}\right) \mid x \in \mathfrak{g}_{1}, x V_{i}^{\perp}=0\right\} \rightarrow \overline{\mathcal{O}}_{2^{i} 1^{2 n-2 i}} .
$$

The $v_{i}$ 's are Reeder's resolutions for $\overline{\mathcal{O}}_{2^{i} 1^{2 n-2 i}}[\mathrm{R}]$. Note that $\mathcal{O}_{2^{j} 1^{2 n-2 j}} \subset \overline{\mathcal{O}}_{2^{i} 1^{2 n-2 i}}$ if and only if $j \leq i$.

Lemma 2.1. Let $x_{j} \in \mathcal{O}_{2^{j} 1^{2 n-2 j}}, j \leq i$. We have

$$
v_{i}^{-1}\left(x_{j}\right) \cong \operatorname{OGr}(i-j, 2 n-2 j) \text {. }
$$

Furthermore, the component group $A_{K}\left(x_{j}\right):=Z_{K}\left(x_{j}\right) / Z_{K}\left(x_{j}\right)^{0}$ of the centralizer $Z_{K}\left(x_{j}\right)$ acts trivially on $H^{*}\left(v_{i}^{-1}\left(x_{j}\right), \mathbb{C}\right)$.

Proof. The proof proceeds in the same manner as the proofs of displayed statements CVX1, (7.1) and (7.2)] for the maps denoted there by $\sigma_{i}$.

Making use of Lemma 2.1, one readily checks that for $x_{j} \in \mathcal{O}_{2^{j} 1^{2 n-2 j}}, 2 \operatorname{dim} v_{i}^{-1}\left(x_{j}\right)-$ $\operatorname{codim}_{\overline{\mathcal{O}}_{2^{i}{ }^{2 n-2 i}}} \mathcal{O}_{2^{j} 1^{2 n-2 j}}=(i-j)(2 n-2 i-1)$. Let us write

$$
m_{i j}=(i-j)(2 n-2 i-1) .
$$

It follows from the decomposition theorem and the lemma above that we have the following decomposition

$$
v_{i *} \mathbb{C}[i(2 n-i)] \cong \bigoplus_{j=0}^{i} \bigoplus_{k=0}^{m_{i j}} \mathrm{IC}\left(\overline{\mathcal{O}}_{2^{j} 1^{2 n-2 j}}, \mathbb{C}^{s_{j k}^{i}}\right)[ \pm k]
$$

where $s_{j k}^{i}$ are non-negative integers and $s_{i k}^{i}=\delta_{0, k}$. The fact that all the local systems appearing in this decomposition are trivial follows from the lemma.

In what follows we write $\left(s_{j k}^{i}\right)_{n}$ for the numbers $s_{j k}^{i}$ to indicate that the ambient symmetric pair is $(\mathrm{SL}(2 n), \mathrm{SO}(2 n))$. The lemma below allows us to compute the numbers $\left(s_{j k}^{i}\right)_{n}$ and the stalks of the $\mathrm{IC}\left(\overline{\mathcal{O}}_{2^{i} 1^{2 n-2 i}}, \mathbb{C}\right)$ simultaneously by induction.

Lemma 2.2. We have

(1) $\left(s_{l k}^{i}\right)_{n}=\left(s_{l-j, k}^{i-j}\right)_{n-j}$.

(2) $\mathcal{H}_{x_{j}}^{k} \operatorname{IC}\left(\overline{\mathcal{O}}_{2^{i} 1^{2 n-2 i}}, \mathbb{C}\right)=\mathcal{H}_{0}^{k+t_{j}} \operatorname{IC}\left(\overline{\mathcal{O}}_{2^{i-j} 1^{2 n-2 i}}, \mathbb{C}\right)$, where $x_{j} \in \mathcal{O}_{2^{j} 1^{2 n-2 j}}$ and $t_{j}=j(2 n-j)$.

Proof. In [CVX1, §7.5] we proved this lemma in the odd case, i.e., for the symmetric pair $(\mathrm{SL}(2 n+1), \mathrm{SO}(2 n+1))$. This argument can be readily adapted to the even case $(\mathrm{SL}(2 n), \mathrm{SO}(2 n))$ we consider in this paper. 
2.3. Fourier transforms of $\operatorname{IC}\left(\overline{\mathcal{O}}_{2^{i} 1^{2 n-2 i}}, \mathbb{C}\right)$. Let $\mathfrak{F}: \mathrm{D}_{K}\left(\mathfrak{g}_{1}\right) \rightarrow \mathrm{D}_{K}\left(\mathfrak{g}_{1}\right)$ be the Fourier transform where we have identified $\mathfrak{g}_{1}$ with $\mathfrak{g}_{1}^{*}$ via a $K$-invariant non-degenerate bilinear form on $\mathfrak{g}_{1}$. Then $\mathfrak{F}$ induces an equivalence of categories $\operatorname{Perv}_{K}\left(\mathfrak{g}_{1}\right) \rightarrow \operatorname{Perv}_{K}\left(\mathfrak{g}_{1}\right)$, where $\operatorname{Perv}_{K}\left(\mathfrak{g}_{1}\right)$ is the category of $K$-equivariant perverse sheaves on $\mathfrak{g}_{1}$. In this subsection we study the Fourier transforms of $\mathrm{IC}\left(\overline{\mathcal{O}}_{2^{i} 1^{2 n-2 i}}, \mathbb{C}\right), i \in[0, n-1]$.

Let us choose a Cartan subspace $\mathfrak{a}$ of $\mathfrak{g}_{1}$ such that it consists of diagonal matrices. Let $\mathfrak{a}^{r s}=\mathfrak{a} \cap \mathfrak{g}^{r s}$ denote the set of regular semisimple elements in $\mathfrak{a}$. For $a \in \mathfrak{a}$ with diagonal entries $a_{1}, \ldots, a_{2 n}$, we write $a=\left(a_{1}, \ldots, a_{2 n}\right)$. Thus $a=\left(a_{1}, \ldots, a_{2 n}\right) \in \mathfrak{a}^{r s}$ if and only if $a_{i} \neq a_{j}$ for $i \neq j$.

Consider the family $C \rightarrow \mathfrak{g}_{1}^{r s}$ whose fiber over $\gamma \in \mathfrak{g}_{1}^{r s}$ is the hyperelliptic curve $C_{\gamma}$ with affine equation $y^{2}=\operatorname{det}(t \cdot \mathrm{id}-\gamma)$. The family above is constant on $K$-orbits and descends to the universal family $\bar{C} \rightarrow \mathfrak{g}_{1}^{r s} / / K=\mathfrak{a}^{r s} / S_{2 n}$ of hyperelliptic curves of genus $n-1$ : to each $a=\left(a_{1}, \ldots, a_{2 n}\right) \in \mathfrak{a}^{r s}$ we associate the hyperelliptic curve $\bar{C}_{a}$ over $\mathbb{P}^{1}$ which ramifies at $\left\{a_{1}, \ldots, a_{2 n}\right\}$. The family $C \rightarrow \mathfrak{g}_{1}^{r s}$ gives us a monodromy representation

$$
\pi_{1}^{K}\left(\mathfrak{g}_{1}^{r s}, \gamma\right) \rightarrow \pi_{1}\left(\mathfrak{g}_{1}^{r s} / / K, \gamma\right)=B_{2 n} \rightarrow \operatorname{Sp}\left(H^{1}\left(C_{\gamma}, \mathbb{C}\right)\right)
$$

Note that, by [A] (see also [KS]) this monodromy representation has a Zariski dense image. From this we get a monodromy representation on the cohomology of the Jacobian of $C_{\gamma}$ which we break into primitive parts:

$$
\pi_{1}^{K}\left(\mathfrak{g}_{1}^{r s}, \gamma\right) \rightarrow \operatorname{Sp}\left(H^{i}\left(\operatorname{Jac}\left(C_{\gamma}\right), \mathbb{C}\right)_{\operatorname{prim}}\right) \cong \operatorname{Sp}\left(\left(\wedge^{i} H^{1}\left(C_{\gamma}, \mathbb{C}\right)\right)_{\operatorname{prim}}\right), \quad i \in[1, n-1]
$$

Associated to this representation $W_{i}$ we obtain an irreducible $K$-equivarant local system $\mathcal{W}_{i}$ on $\mathfrak{g}_{1}^{r s}$. Note that the part $A[2]$ of $\pi_{1}^{K}\left(\mathfrak{g}_{1}^{r s}\right)$ acts trivially on $\mathcal{W}_{i}$. It is clear that we have $\mathcal{W}_{i} \not \mathcal{W}_{j}$ for $i \neq j$ and

$$
\operatorname{dim} W_{i}=\left(\begin{array}{c}
2 n-2 \\
i
\end{array}\right)-\left(\begin{array}{c}
2 n-2 \\
i-2
\end{array}\right) .
$$

Proposition 2.3. We have

$$
\mathfrak{F}\left(\mathrm{IC}\left(\overline{\mathcal{O}}_{2^{i} 1^{2 n-2 i}}, \mathbb{C}\right)\right) \cong \mathrm{IC}\left(\mathfrak{g}_{1}, \mathcal{W}_{i}\right), i \in[0, n-1]
$$

where $\mathcal{W}_{i}$ are irreducible $K$-equivariant local systems on $\mathfrak{g}_{1}^{\text {rs }}$ defined above and $\mathcal{W}_{0}=\mathbb{C}$ is the trivial local system.

Proof. Consider the map $v_{n-1}$ defined in (2.1). Let us write $v=v_{n-1}$ and

$$
E=\left\{\left(x, 0 \subset V_{n-1} \subset V_{n-1}^{\perp} \subset \mathbb{C}^{2 n}\right) \mid x \in \mathfrak{g}_{1}, x V_{n-1}^{\perp}=0\right\} .
$$

Then $v: E \rightarrow \overline{\mathcal{O}}_{2^{n-1} 1^{2}}$ is the natural projection. It follows from (2.2) that we have

$$
v_{*} \mathbb{C}\left[n^{2}-1\right] \cong \bigoplus_{j=0}^{n-1} \bigoplus_{k=0}^{n-j-1} \operatorname{IC}\left(\overline{\mathcal{O}}_{2^{j} 1^{2 n-2 j}}, \mathbb{C}^{\left(s_{j k}^{n-1}\right)_{n}}\right)[ \pm k] .
$$

Let $E^{\perp}$ be the orthogonal complement of $E$ in the trivial bundle $\mathfrak{g}_{1} \times X \rightarrow X$, where $X=\left\{V_{n-1} \mid 0 \subset V_{n-1} \subset V_{n-1}^{\perp} \subset \mathbb{C}^{2 n}\right\} \cong \operatorname{OGr}(n-1,2 n)$, i.e.,

$$
E^{\perp}=\left\{\left(x, 0 \subset V_{n-1} \subset V_{n-1}^{\perp} \subset \mathbb{C}^{2 n}\right) \mid x \in \mathfrak{g}_{1}, x V_{n-1} \subset V_{n-1}^{\perp}\right\} .
$$


Consider the natural projection map $\check{v}: E^{\perp} \rightarrow \mathfrak{g}_{1}$. By functoriality of the Fourier transform, we obtain:

$$
\mathfrak{F}\left(v_{*} \mathbb{C}[-]\right) \cong \check{v}_{*} \mathbb{C}[-]
$$

We show that

$$
\begin{aligned}
\check{v}_{*} \mathbb{C}[-]= & \left(\bigoplus_{k=0}^{\left[\frac{n-1}{2}\right]} \operatorname{IC}\left(\mathfrak{g}_{1}, \bigoplus_{j=0}^{k} \mathcal{W}_{2 j}\right)[ \pm(n-1-2 k)]\right) \\
& \oplus\left(\bigoplus_{k=0}^{\left[\frac{n-1}{2}\right]-1} \operatorname{IC}\left(\mathfrak{g}_{1}, \bigoplus_{j=0}^{k} \mathcal{W}_{2 j+1}\right)[ \pm(n-2-2 k)]\right) \oplus \cdots
\end{aligned}
$$

where the omitted term $\cdots$ consists of IC complexes that are supported on proper subsets of $\mathfrak{g}_{1}$. Using (2.4) and comparing (2.3) with (2.5), we conclude that the proposition holds. It remains to prove (2.5).

For any $\gamma \in \mathfrak{g}_{1}^{r s}$ let

$$
F_{\gamma}=\check{v}^{-1}(\gamma) \cong\left\{0 \subset V_{n-1} \subset V_{n-1}^{\perp} \subset \mathbb{C}^{2 n} \mid \gamma V_{n-1} \subset V_{n-1}^{\perp}\right\},
$$

which is the Fano variety of $(n-2)$-planes in the smooth complete intersection of the two quadrics $Q=0$ and $Q_{\gamma}=\langle\gamma-,-\rangle_{Q}=0$. Recall the hyperelliptic curve $C_{\gamma}$. According to $[\mathrm{Re}, \mathrm{Do}, \mathrm{W}]$, there is a canonical action of $\operatorname{Jac}\left(C_{\gamma}\right)$ on $F_{\gamma}$ such that $F_{\gamma}$ becomes a $\operatorname{Jac}\left(C_{\gamma}\right)$ torsor under this action. This action extends to families: as $\gamma$ varies over $\mathfrak{g}_{1}^{r s}$, we obtain a $\operatorname{Jac}(C)$-torsor $\left.\check{v}\right|_{\check{v}^{-1}\left(\mathfrak{g}_{1}^{r s}\right)}: F \rightarrow \mathfrak{g}_{1}^{r s}$ of Fano varieties of $(n-2)$-planes in complete intersections of two quadrics. By taking cohomologies of fibers, the families $\operatorname{Jac}(C)$ and $F$ give rise to local systems on $\mathfrak{g}_{1}^{r s}$. We claim that those local systems coincide, i.e., that

$$
\text { The } \pi_{1}^{K}\left(\mathfrak{g}_{1}^{r s}, \gamma\right) \text {-representations } H^{i}\left(\operatorname{Jac}\left(C_{\gamma}\right), \mathbb{C}\right) \text { and } H^{i}\left(F_{\gamma}, \mathbb{C}\right)
$$

are canonically isomorphic.

As $F$ does not appear to have a natural section we argue as follows. According to [W] there is a canonical involution $\sigma$ on $F_{\gamma}$ compatible with the inversion map on $\operatorname{Jac}\left(C_{\gamma}\right)$. Let $F_{\gamma}^{\sigma}$ be the set of $\sigma$-fixed points on $F_{\gamma}$. Then $F_{\gamma}^{\sigma}$ is naturally a $\operatorname{Jac}\left(C_{\gamma}\right)[2]$-torsor. Thus, the $\operatorname{Jac}(C)$-torsor $F$ gives rise to a $\operatorname{Jac}(C)[2]$-torsor $F^{\sigma} \rightarrow \mathfrak{g}_{1}^{r s}$ consisting of $\sigma$ fixed points on $F$. We note that there is a canonical isomorphism

$$
\left(\operatorname{Jac}(C) \times_{\mathfrak{g}_{1}^{r s}} F^{\sigma}\right) / \operatorname{Jac}(C)[2] \cong F
$$

where $\operatorname{Jac}(C)[2]$ acts on $\operatorname{Jac}(C) \times_{\mathfrak{g}_{1}^{r s}} F^{\sigma}$ via the diagonal action. Moreover, arguing as in [CVX1, Lemma 5.5], we obtain (2.6). We then conclude that

$$
\left(\left.R^{i} \check{v}_{*}\right|_{\mathfrak{g}_{1}^{r s}}\right)_{\text {prim }} \cong \mathcal{W}_{i}, i \in[1, n-1]
$$

Thus (2.5) follows. 


\section{Cohomology of Fano varieties}

In this section we compute the cohomology of the Fano varieties $\mathrm{Fano}_{k}$ of $k$-planes contained in the smooth complete intersection of two quadrics in $\mathbb{P}^{2 n-1}$, making use of the results in $\$ 2$.

3.1. Fano varieties. Consider the natural projection maps

$$
\check{v}_{i}:\left\{\left(x, 0 \subset V_{i} \subset V_{i}^{\perp} \subset \mathbb{C}^{2 n}\right) \mid x \in \mathfrak{g}_{1}, x V_{i} \subset V_{i}^{\perp}\right\} \rightarrow \mathfrak{g}_{1} .
$$

For $\gamma \in \mathfrak{g}_{1}^{r s}$, the fiber $\check{v}_{i}^{-1}(\gamma)$ can be identified with the Fano variety Fano $_{i-1}$ of $(i-1)$ planes contained in the smooth complete intersection of the two quadrics $Q=0$ and $Q_{\gamma}=$ $\langle\gamma-,-\rangle_{Q}=0$ in $\mathbb{P}^{2 n-1}$. It is easy to see that

$$
d_{i}:=\operatorname{dim} \mathrm{Fano}_{i-1}=\operatorname{dim} \check{v}_{i}^{-1}(\gamma)=i(2 n-2 i-1) .
$$

Consider $\pi_{i}=\left.\check{v}_{i}\right|_{\check{v}_{i}^{-1}\left(\mathfrak{g}_{1}^{r s}\right)}$, which is a smooth family of Fano varieties, and consider the corresponding local systems $R^{k} \pi_{i *} \mathbb{C}$. Utilizing functoriality of the Fourier transform, just as in (2.4), we have:

$$
\mathfrak{F}\left(\check{v}_{i *} \mathbb{C}[-]\right) \cong v_{i *} \mathbb{C}[-] .
$$

Together with (2.2), this implies that

$$
\begin{aligned}
& \mathfrak{F}\left(\check{v}_{i *} \mathbb{C}[-]\right) \cong \bigoplus_{k=0}^{2 d_{i}} \mathfrak{F}\left(\mathrm{IC}\left(\mathfrak{g}_{1}, R^{k} \pi_{i *} \mathbb{C}\right)\left[-k+d_{i}\right]\right) \\
& \cong \bigoplus_{j=0}^{i} \bigoplus_{k=0}^{(i-j)(2 n-2 i-1)} \mathrm{IC}\left(\overline{\mathcal{O}}_{2^{j} 1^{2 n-2 j}}, \mathbb{C}^{\left(s_{j k}^{i}\right)_{n}}\right)[ \pm k] .
\end{aligned}
$$

Hence we see that $\mathfrak{F}\left(\mathrm{IC}\left(\mathfrak{g}_{1}, R^{k} \pi_{i *} \mathbb{C}\right)\right)$ is supported on $\overline{\mathcal{O}}_{2^{i} 1^{2 n-2 i}}$, and has the form

$$
\mathfrak{F}\left(\mathrm{IC}\left(\mathfrak{g}_{1}, R^{k} \pi_{i *} \mathbb{C}\right)\right) \cong \bigoplus_{j=0}^{i} \mathrm{IC}\left(\overline{\mathcal{O}}_{2^{j} 1^{2 n-2 j}}, \mathbb{C}^{\left(s_{j,\left|d_{i}-k\right|}^{i}\right)_{n}}\right)
$$

It follows from the discussion above and Proposition 2.3 that the cohomology of the Fano variety $\mathrm{Fano}_{i-1}$ is described as follows

$$
H^{k}\left(\mathrm{Fano}_{i-1}, \mathbb{C}\right) \cong \bigoplus_{j=0}^{i}\left(s_{j,\left|d_{i}-k\right|}^{i}\right)_{n} W_{j}
$$

It remains to determine the numbers $\left(s_{j k}^{i}\right)_{n}$.

3.2. The numbers $\left(s_{j k}^{i}\right)_{n}$ and stalks for $\operatorname{IC}\left(\mathcal{O}_{2^{i} 1^{2 n-2 i}}, \mathbb{C}\right)$ at 0 . In this subsection, we give explicit formulas for the numbers $\left(s_{j k}^{i}\right)_{n}$ and the dimensions of the stalks $\mathcal{H}_{0}^{k} \mathrm{IC}\left(\mathcal{O}_{2^{i} 1^{2 n-2 i}}, \mathbb{C}\right)$ in terms of their generating functions. Note first that Lemma 2.2 (1) implies that

$$
\left(s_{j k}^{i}\right)_{n}=\left(s_{0, k}^{i-j}\right)_{n-j} \text { for } j \geq 1 .
$$

Since $\left(s_{i, k}^{i}\right)_{n}=\delta_{0, k}$, it suffices to study the numbers $\left(s_{0, k}^{i}\right)_{n}$. 
For $0 \leq j \leq n-1$, let us define

$$
h_{j}^{(n)}(q):=(-1)^{j} q^{j(2 n-j)} \sum_{k}(-1)^{k}\left(\operatorname{dim} \mathcal{H}_{0}^{k} \operatorname{IC}\left(\overline{\mathcal{O}}_{2^{j} 1^{2 n-2 j}}, \mathbb{C}\right)\right) q^{k},
$$

and

$$
P_{j}^{(n)}(q):=(-1)^{j} q^{j(2 n-2 j-1)} \sum_{k=0}^{j(2 n-2 j-1)}(-1)^{k}\left(s_{0 k}^{j}\right)_{n} q^{ \pm k} .
$$

Note that $h_{0}^{(n)}(q)=P_{0}^{(n)}(q)=1$ and

$$
\text { For } j \geq 1 \text {, the function } h_{j}^{(n)}(q) \text { (resp. } P_{j}^{(n)}(q) \text { ) is a polynomial in } q \text { with }
$$
degree no greater than $j(2 n-j)-1($ resp. $2 j(2 n-2 j-1))$.

$$
\text { The coefficients of } q^{j(2 n-2 j-1)+k} \text { and } q^{j(2 n-2 j-1)-k} \text { in } P_{j}^{(n)}(q) \text { are equal for all } k \geq 1 \text {. }
$$

The statement above for $h_{j}^{(n)}(q), j \geq 1$, follows from the fact that $\mathcal{H}_{0}^{k} \mathrm{IC}\left(\overline{\mathcal{O}}_{2^{j} 1^{2 n-2 j}}, \mathbb{C}\right)$ is non-zero only if $-\operatorname{dim} \mathcal{O}_{2^{j} 1^{2 n-2 j-1}}=-j(2 n-j) \leq k \leq-1$.

For $0<i \leq k$, let us write $g_{i, k}(q)$ for the Poincare polynomial of the Grassmannian variety of $i$-dimensional subspaces in $\mathbb{C}^{k}$, i.e.,

$$
g_{i, k}(q)=\frac{\prod_{s=k-i+1}^{k}\left(1-q^{2 s}\right)}{\prod_{s=1}^{i}\left(1-q^{2 s}\right)} .
$$

Let us also define

$$
g_{0, k}(q)=1 \text { and } g_{i, k}(q)=0 \text { for } i<0 .
$$

Proposition 3.1. We have

$$
\begin{gathered}
h_{j}^{(n)}(q)=\sum_{k=0}^{[j / 2]}\left(\left(\prod_{s=1}^{j-2 k}\left(1+q^{2 n-2 s}\right)\right) \cdot\left(g_{k, n-j+2 k-1}\left(q^{2}\right)-g_{k-1, n-j+2 k-1}\left(q^{2}\right)\right)\right), \\
P_{j}^{(n)}(q)=g_{j, 2 n-1-j}(q) .
\end{gathered}
$$

Proof. Note that $v_{i}^{-1}(0) \cong \operatorname{OGr}(i, 2 n)$. Let us write

$$
o g_{j, 2 n}(q)=\sum_{k=0}^{r_{j}}(-1)^{k} \operatorname{dim} H^{k}(\operatorname{OGr}(j, 2 n), \mathbb{C}) q^{k},
$$

where $r_{j}=2 \operatorname{dim} \operatorname{OGr}(j, 2 n)=j(4 n-3 j-1)$. The polynomials $\operatorname{og}_{j, 2 n}(q)$ are well-known, i.e.,

$$
o g_{j, 2 n}(q)=\frac{\left(1-q^{2 n}\right) \prod_{k=n-j}^{n-1}\left(1-q^{4 k}\right)}{\left(1-q^{2(n-j)}\right) \prod_{k=1}^{j}\left(1-q^{2 k}\right)}
$$


Taking stalks $\mathcal{H}_{0}^{k}$ on both sides of the equation (2.2), we obtain that

$$
\begin{gathered}
(-1)^{i} \operatorname{og}_{i, 2 n}(q) q^{-i(2 n-i)}= \\
\sum_{j=0}^{i}\left(\sum_{k}(-1)^{k}\left(\operatorname{dim} \mathcal{H}_{0}^{k} \operatorname{IC}\left(\overline{\mathcal{O}}_{2^{j} 1^{2 n-2 j}}, \mathbb{C}\right)\right) q^{k}\right) \cdot\left(\sum_{k=0}^{(i-j)(2 n-2 i-1)}(-1)^{k}\left(s_{j k}^{i}\right)_{n} q^{ \pm k}\right) .
\end{gathered}
$$

Making use of (3.3), the equation (3.12) can be written in terms of the functions defined in (3.4) and (3.5) as

$$
o g_{i, 2 n}(q)=\sum_{j=0}^{i} q^{(i-j)(i-j+1)} h_{j}^{(n)}(q) P_{i-j}^{(n-j)}(q)
$$

Observe that

The equations (3.13) determine the polynomials $h_{i}^{(n)}(q)$ and $P_{i}^{(n)}(q)$ uniquely given that $h_{i}^{(n)}(q)$ and $P_{i}^{(n)}(q)$ satisfy (3.6) and (3.7) .

In fact, this follows from a simple induction argument. Given that $P_{i}^{(n)}(q)$ satisfy (3.7), it suffices to determine the coefficient of $q^{k}$ in $P_{i}^{(n)}(q)$ for $k \geq i(2 n-2 i-1)$, or equivalently, the coefficient of $q^{k}$ in $q^{i(i+1)} P_{i}^{(n)}(q)$ for $k \geq i(2 n-i)$. By induction, we can assume that in (3.13), $h_{j}^{(m)}(q)$ and $P_{j}^{(m)}(q)$ are known for all $j<i$ and all $m$. Now the degree of $h_{i}^{(n)}(q)$ is at most $i(2 n-i)-1$. Thus (3.13) determines $P_{i}^{(n)}(q)$ and $h_{i}^{(n)}(q)$ uniquely.

In view of the observation (3.14), the proposition follows from Theorem A.1 and Corollary A.2 in Appendix A. Note that $g_{i, k}(q)=\left[\begin{array}{l}k \\ i\end{array}\right]_{q^{2}}$ in the notation of Definition A.1.

\section{Corollary 3.2. We have}

(1) $h_{i}^{(n)}(q) \in \mathbb{Z}_{\geq 0}\left[q^{2}\right]$

(2) $\mathcal{H}^{k} \mathrm{IC}\left(\overline{\mathcal{O}}_{2^{i} 1^{2 n-2 i}}, \mathbb{C}\right)=0$ if $k \equiv i-1(\bmod 2)$.

Proof. For (1) it suffices to show that $h_{i}^{(n)}(q) \in \mathbb{Z}\left[q^{2}\right]$. This follows from (3.13) and induction on $i$. See also Corollary A.2 for a direct proof. To prove (2) we observe that (1) implies $\mathcal{H}_{0}^{k} \mathrm{IC}\left(\overline{\mathcal{O}}_{2^{i} 1^{2 n-2 i}}, \mathbb{C}\right)=0$ if $k \equiv i-1(\bmod 2)$. Now the desired claim follows from Lemma 2.2 ,

Remark 3.1. As noted in the introduction, it would be interesting to understand the formula (3.13) in geometric terms. The formula involves doubling certain cohomological degrees and we have been unable to come up with a geometric interpretation for this phenomenon.

Remark 3.2. As discussed in the introduction, the functions $h_{m}^{(n)}(q)$ can be viewed as a symmetric space analogue of Kostka-Foulkes polynomials. In [CVX1, Theorem 7.1, Lemma 8.3] we have shown that in the odd case, i.e., for the symmetric pair $(\mathrm{SL}(2 n+1), \mathrm{SO}(2 n+1))$, 
the local IC on nilpotent orbits of order two are isomorphic to the local IC on nilpotent orbits of order two in $\mathfrak{s p}(2 n)$. In turn, we deduced that the corresponding "Kostka-Foulkes polynomials" are given by the fake degree polynomials, which have a much simpler form.

3.3. Proof of Theorem 1.1. Recall that $d_{i}=\operatorname{dim} \mathrm{Fano}_{i-1}=i(2 n-2 i-1)$ (see (3.1)). Let us write $\bar{\wedge}^{j}=\bar{\wedge}^{j}\left(H^{1}(C, \mathbb{C})\right)=\wedge^{n-1-j}\left(H^{1}(C, \mathbb{C})\right)$. It follows from (3.2) and (3.3) that we have

$$
\begin{aligned}
& H^{d_{n-i}-k}\left(\mathrm{Fano}_{n-i-1}, \mathbb{C}\right) \cong \bigoplus_{j=0}^{n-i}\left(s_{j,|k|}^{n-i}\right)_{n} W_{j} \cong \bigoplus_{j=0}^{n-i}\left(\left(s_{j,|k|}^{n-i}\right)_{n}-\left(s_{j+2,|k|}^{n-i}\right)_{n}\right) \wedge^{j}\left(H^{1}(C, \mathbb{C})\right) \\
& \cong \bigoplus_{j=0}^{n-i}\left(\left(s_{0,|k|}^{n-i-j}\right)_{n-j}-\left(s_{0,|k|}^{n-i-j-2}\right)_{n-j-2}\right) \bar{\wedge}^{n-1-j} \cong \bigoplus_{j=i-1}^{n-1}\left(\left(s_{0,|k|}^{j-i+1}\right)_{j+1}-\left(s_{0,|k|}^{j-i-1}\right)_{j-1}\right) \bar{\wedge}^{j}
\end{aligned}
$$

Here we have used the convention that $\left(s_{0, b}^{a}\right)_{m}=0$ if $a<0$. Thus we have

$$
N_{i}(k, j)=\left(s_{0,|k|}^{j-i+1}\right)_{j+1}-\left(s_{0,|k|}^{j-i-1}\right)_{j-1} .
$$

Using (3.5) and (3.11), we see that

$$
\begin{aligned}
& \left(s_{0,|k|}^{j-i+1}\right)_{j+1} \text { is the coefficient of } q^{k}\left(\text { or } q^{-k}\right) \text { in }(-1)^{j-i+1+k} q^{-(j-i+1)(2 i-1)} g_{j-i+1, i+j}(q), \\
& \left(s_{0,|k|}^{j-i-1}\right)_{j-1} \text { is the coefficient of } q^{k}\left(\text { or } q^{-k}\right) \text { in }(-1)^{j-i+1+k} q^{-(j-i-1)(2 i-1)} g_{j-i-1, i+j-2}(q) .
\end{aligned}
$$

Using (3.8) and (3.9) , one readily checks that $N_{i}(k, j)$ is the coefficient of $q^{k}$ (or $\left.q^{-k}\right)$ in

$$
(-1)^{j-i+1+k} q^{-(j-i+1)(2 i-1)}\left(1-q^{4 j}\right) \frac{\prod_{l=j-i+2}^{i+j-2}\left(1-q^{2 l}\right)}{\prod_{l=1}^{2 i-2}\left(1-q^{2 l}\right)} .
$$

Note that such a coefficient is nonzero only if $k \equiv j-i+1(\bmod 2)$; in the latter case $(-1)^{j-i-1+k}=1$. This proves the theorem as $n=g+1$.

Remark 3.3. For $i=2$, the formula in Theorem 1.1 coincides with the formula in [N, Theorem $1]$.

Example 3.4. The cohomology of Fano ${ }_{1}$, the Fano variety of lines in the smooth complete intersection of two quadrics in $\mathbb{P}^{2 n-1}$ can be described as follows:

$$
\begin{gathered}
H^{8 n-20-k}\left(\mathrm{Fano}_{1}, \mathbb{C}\right) \cong H^{k}\left(\mathrm{Fano}_{1}, \mathbb{C}\right) \\
\cong \begin{cases}\mathbb{C}^{\left[\frac{m+2}{2}\right]} & \text { if } k=2 m \text { and } 0 \leq m \leq 2 n-6, \\
H^{1}(C, \mathbb{C}) & \text { if } k=2 m+1 \text { and } n-3 \leq m \leq 2 n-6, \\
\mathbb{C}^{n-3} \oplus \wedge^{2}\left(H^{1}(C, \mathbb{C})\right) & \text { if } k=4 n-10 .\end{cases}
\end{gathered}
$$




\section{Appendix A. The functions $h_{m}^{(n)}(q)$}

\section{by Dennis Stantor 1}

The main results for $h_{m}^{(n)}(q)$ are given in Theorem A.1 and Corollary A.2,

Definition A.1. For non-negative integers $n$ and $k$ let

$$
(A ; q)_{n}=\prod_{k=0}^{n-1}\left(1-A q^{k}\right), \quad\left[\begin{array}{l}
n \\
k
\end{array}\right]_{q}=\frac{\left(q^{n} ; q^{-1}\right)_{k}}{(q ; q)_{k}} .
$$

Note that the $q$-binomial coefficient $\left[\begin{array}{l}n \\ k\end{array}\right]_{q}$ is known to be a polynomial in $q$ of degree $k(n-k)$ with non-negative coefficients, see [An2, Theorem 3.2, p. 35].

Definition A.2. For $0 \leq k \leq n-1$ let

$$
\operatorname{og}_{k, 2 n}(q)=\frac{\left(q^{4(n-k)} ; q^{4}\right)_{k}}{\left(q^{2} ; q^{2}\right)_{k}} \frac{1-q^{2 n}}{1-q^{2(n-k)}} .
$$

Definition A.3. For $n \geq 1$ and $0 \leq j \leq n-1$ let $h_{j}^{(n)}(q)$ for $0 \leq j \leq n-1$ be defined recursively by the $n$ equations

$$
\operatorname{og}_{k, 2 n}(q)=\sum_{j=0}^{k} q^{(k-j)(k-j+1)} h_{j}^{(n)}(q)\left[\begin{array}{c}
2 n-1-k-j \\
k-j
\end{array}\right]_{q^{2}}, \quad 0 \leq k \leq n-1 .
$$

An explicit formula for $h_{m}^{(n)}(q)$ is the main result. The proof is given at the end of the Appendix.

Theorem A.1. If $0 \leq m \leq n-1$, then

$$
\begin{aligned}
h_{m}^{(n)}(q) & =\sum_{k=0}^{[m / 2]}\left(-q^{2 n-2} ; q^{-2}\right)_{m-2 k}\left[\begin{array}{c}
n-m+2 k \\
k
\end{array}\right]_{q^{4}} \frac{1-q^{4(n-m)}}{1-q^{4(n-m+2 k)}} q^{4 k} \\
& =\sum_{k=0}^{[m / 2]}\left(-q^{2 n-2} ; q^{-2}\right)_{m-2 k}\left(\left[\begin{array}{c}
n-m+2 k-1 \\
k
\end{array}\right]_{q^{4}}-\left[\begin{array}{c}
n-m+2 k-1 \\
k-1
\end{array}\right]_{q^{4}}\right),
\end{aligned}
$$

where

$$
\left[\begin{array}{c}
n-m-1 \\
-1
\end{array}\right]_{q^{4}}=0 .
$$

Remark A.1. Note that Theorem A.1 implies the recurrence

$$
h_{m}^{(n)}(q)=\left\{\begin{array}{l}
\left(1+q^{2 n-2}\right) h_{m-1}^{(n-1)}(q), \quad \text { if } m \geq 1 \text { is odd, } \\
\left(1+q^{2 n-2}\right) h_{m-1}^{(n-1)}(q)+\left[\begin{array}{c}
n-1 \\
m / 2
\end{array}\right]_{q^{4}}-\left[\begin{array}{c}
n-1 \\
m / 2-1
\end{array}\right]_{q^{4}}, \quad \text { if } m \geq 2 \text { is even. }
\end{array}\right.
$$

\footnotetext{
${ }^{1}$ School of Mathematics, University of Minnesota, USA. E-mail: stant001@umn.edu.
} 
The polynomiality and positivity of $h_{m}^{(n)}(q)$ follows from Theorem A.1.

Corollary A.2. If $0 \leq m \leq n-1$, then $h_{m}^{(n)}(q)$ is a polynomial in $q$ of degree $m(2 n-m-1)$ with non-negative integer coefficients.

Proof. In fact we show that the $k^{\text {th }}$ term in the sum of Theorem A.1 is a non-negative polynomial in $q$ of degree $m(2 n-m-1)-2 k$.

The factor

$$
\left(-q^{2 n-2} ; q^{-2}\right)_{m-2 k}=\prod_{j=0}^{m-2 k-1}\left(1+q^{2 n-2-2 j}\right)
$$

is a non-negative polynomial in $q$ of degree $(2 n-2)(m-2 k)-2\left(\begin{array}{c}m-2 k \\ 2\end{array}\right)$.

The factor

$$
\left[\begin{array}{c}
n-m+2 k-1 \\
k
\end{array}\right]_{q^{4}}-\left[\begin{array}{c}
n-m+2 k-1 \\
k-1
\end{array}\right]_{q^{4}}
$$

is a non-negative polynomial in $q^{4}$ of degree $k(n-m+k-1)$. This difference is known to be non-negative, see [An1. It is also the fake degree polynomial $f^{\lambda}\left(q^{4}\right)$, for a partition $\lambda=(n-m-1+k, k)$ with 2 rows. It may be written as the generating function for the major index of standard Young tableaux of shape $\lambda$, see [St, Corollary 7.21.5, p. 376].

Since

$$
(2 n-2)(m-2 k)-2\left(\begin{array}{c}
m-2 k \\
2
\end{array}\right)+4 k(n-m+k-1)=m(2 n-m-1)-2 k,
$$

the $k^{\text {th }}$ term has degree $m(2 n-m-1)-2 k$ and is non-negative.

The proof of Theorem A.1 is in two steps. First, an explicit formula for $h_{m}^{(n)}(q)$ is found by inverting the matrix in Definition A.3, see Proposition A.4. Next a basic hypergeometric transformation, Proposition A.6, is applied to obtain Theorem A.1.

First we have a matrix inverse result which is [GS, Theorem 3.2].

Proposition A.3. Suppose that

$$
\beta_{k}=\sum_{j=0}^{k} \frac{\alpha_{j}}{(Q ; Q)_{k-j}(A Q ; Q)_{k+j}}, \quad 0 \leq k \leq n-1 .
$$

Then for $0 \leq m \leq n-1$,

$$
\alpha_{m}=\sum_{k=0}^{m}(-1)^{m+k} \beta_{k} \frac{(A Q ; Q)_{m+k-1}}{(Q ; Q)_{m-k}}\left(1-A Q^{2 m}\right) Q^{\left(\begin{array}{c}
m-k \\
2
\end{array}\right)} \text {. }
$$


We apply Proposition A.3 to Definition A.3. Fix $n \geq 1$. Rewrite Definition A.3 as

$$
\frac{(-1)^{k} \operatorname{og}_{k, 2 n}(q)}{\left(q^{4 n-2} ; q^{-2}\right)_{2 k}}=\sum_{j=0}^{k} \frac{(-1)^{j} h_{j}^{(n)}(q)}{\left(q^{-2} ; q^{-2}\right)_{k-j}\left(q^{4 n-2} ; q^{-2}\right)_{k+j}}, 0 \leq k \leq n-1
$$

So if

$$
Q=q^{-2}, \quad A=q^{4 n}, \quad \beta_{k}=\frac{(-1)^{k} o g_{k, 2 n}(q)}{\left(q^{4 n-2} ; q^{-2}\right)_{2 k}}, \quad \alpha_{j}=(-1)^{j} h_{j}^{(n)}(q)
$$

we can apply Proposition A.3 to solve for $h_{m}^{(n)}(q)$.

Proposition A.4. If $0 \leq m \leq n-1$, then

$$
\begin{aligned}
h_{m}^{(n)}(q) & =\sum_{k=0}^{m} \frac{o g_{k, 2 n}(q)}{\left(q^{4 n-2} ; q^{-2}\right)_{2 k}} \frac{\left(q^{4 n-2} ; q^{-2}\right)_{m+k-1}}{\left(q^{-2} ; q^{-2}\right)_{m-k}}\left(1-q^{4 n-4 m}\right) q^{-2\left(\begin{array}{c}
m-k \\
2
\end{array}\right)} \\
& =C_{m} \sum_{k=0}^{m} \frac{\left(q^{2 m} ; q^{-2}\right)_{k}}{\left(q^{-2} ; q^{-2}\right)_{k}} \frac{\left(q^{4 n-2 m} ; q^{-2}\right)_{k}}{\left(q^{4 n-2} ; q^{-4}\right)_{k}} \frac{\left(1-q^{2 n}\right)}{\left(1-q^{2(n-k)}\right)} q^{-k^{2}-3 k}
\end{aligned}
$$

where

$$
C_{m}=\frac{\left(q^{4 n-2} ; q^{-2}\right)_{m-1}}{\left(q^{2} ; q^{2}\right)_{m}}\left(1-q^{4 n-4 m}\right) q^{2 m}(-1)^{m}
$$

For the final step in the proof of Theorem A.1, we will need a transformation, Corollary A.6. The next result is used to prove Corollary A.6, and is a quadratic transformation of a basic hypergeometric series.

Proposition A.5. As formal power series in $x$,

$$
\sum_{n=0}^{\infty} \frac{\left(D^{2} ; q\right)_{n}}{(q ; q)_{n}} \frac{\left(R / q ; q^{2}\right)_{n}}{(R / q ; q)_{n}} x^{n}=\sum_{k=0}^{\infty} \frac{\left(D^{2} ; q\right)_{2 k}}{\left(q^{2} ; q^{2}\right)_{k}\left(R ; q^{2}\right)_{k}} \frac{\left(D^{2} x q^{2 k} ; q\right)_{\infty}}{(x ; q)_{\infty}} q^{k(2 k-2)} R^{k} x^{2 k}
$$

Proof. We find the coefficient of $x^{n}$ on the right side, and show that it equals the coefficient of $x^{n}$ on the left side. Use the $q$-binomial theorem [An2, Theorem 2.1, p. 17] to expand

$$
\frac{\left(D^{2} x q^{2 k} ; q\right)_{\infty}}{(x ; q)_{\infty}}=\sum_{j=0}^{\infty} \frac{\left(D^{2} q^{2 k} ; q\right)_{j}}{(q ; q)_{j}} x^{j}
$$


So the coefficient of $x^{n}$ on the right side is

$$
\begin{aligned}
& \sum_{k=0}^{[n / 2]} \frac{\left(D^{2} ; q\right)_{2 k}}{\left(q^{2} ; q^{2}\right)_{k}\left(R ; q^{2}\right)_{k}} q^{k(2 k-2)} R^{k} \frac{\left(D^{2} q^{2 k} ; q\right)_{n-2 k}}{(q ; q)_{n-2 k}} \\
& \quad=\frac{\left(D^{2} ; q\right)_{n}}{(q ; q)_{n}} \sum_{k=0}^{[n / 2]} \frac{\left(q^{n} ; q^{-1}\right)_{2 k}}{\left(q^{2} ; q^{2}\right)_{k}\left(R ; q^{2}\right)_{k}} q^{k(2 k-2)} R^{k} \\
& \quad=\frac{\left(D^{2} ; q\right)_{n}}{(q ; q)_{n}} \sum_{k=0}^{[n / 2]} \frac{\left(q^{n} ; q^{-2}\right)_{k}\left(q^{n-1} ; q^{-2}\right)_{k}}{\left(q^{-2} ; q^{-2}\right)_{k}\left(1 / R ; q^{-2}\right)_{k}} q^{-2 k} \\
& \quad=\frac{\left(D^{2} ; q\right)_{n}}{(q ; q)_{n}} \frac{\left(R / q ; q^{2}\right)_{n}}{(R / q ; q)_{n}}
\end{aligned}
$$

where we have used the $q$-Vandermonde theorem [GR, (II.6), p. 354], to evaluate the last sum.

The transformation we need is a corollary of Proposition A.5, and is a $q$-analogue of a result of Bailey $[\mathrm{B},(5.41)]$.

Corollary A.6. If $m$ is a non-negative integer, then

$$
\begin{aligned}
& \sum_{k=0}^{[m / 2]} \frac{\left(D^{2} ; q\right)_{2 k}}{\left(q^{2} ; q^{2}\right)_{k}\left(R ; q^{2}\right)_{k}} q^{k(2 k-2)} R^{k} \frac{\left(D^{2} q^{2 k} / B ; q\right)_{m-2 k}}{(q ; q)_{m-2 k}} B^{m-2 k} \\
& \quad=\sum_{s=0}^{m} \frac{\left(D^{2} ; q\right)_{m-s}}{(q ; q)_{m-s}} \frac{\left(R / q ; q^{2}\right)_{m-s}}{(R / q ; q)_{m-s}} \frac{(1 / B ; q)_{s}}{(q ; q)_{s}} B^{s} \\
& \quad=\frac{\left(D^{2} ; q\right)_{m}}{(q ; q)_{m}} \frac{\left(R / q ; q^{2}\right)_{m}}{(R / q ; q)_{m}} \sum_{s=0}^{m} \frac{\left(q^{-m} ; q\right)_{s}}{\left(q^{1-m} / D^{2} ; q\right)_{s}} \frac{\left(q^{2-m} / R ; q\right)_{s}}{\left(q^{3-2 m} / R ; q^{2}\right)_{s}} \frac{(1 / B ; q)_{s}}{(q ; q)_{s}}\left(\frac{B q^{2-m}}{D^{2}}\right)^{s} q^{\left(\begin{array}{c}
s \\
2
\end{array}\right)}
\end{aligned}
$$

Proof. Multiply both sides of Proposition A.5 by $(x ; q)_{\infty} /(B x ; q)_{\infty}$ and equate coefficients of $x^{m}$, using

$$
\frac{(x ; q)_{\infty}}{(B x ; q)_{\infty}}=\sum_{j=0}^{\infty} \frac{(1 / B ; q)_{j}}{(q ; q)_{j}}(B x)^{j}, \quad \frac{\left(D^{2} x q^{2 k} ; q\right)_{\infty}}{(B x ; q)_{\infty}}=\sum_{j=0}^{\infty} \frac{\left(D^{2} q^{2 k} / B ; q\right)_{j}}{(q ; q)_{j}}(B x)^{j} .
$$

The last equality uses

$$
(A ; Q)_{m-s}=\frac{(A ; Q)_{m}}{\left(Q^{1-m} / A ; Q\right)_{s}\left(-A Q^{m-1}\right)^{s} Q^{-\left(\begin{array}{c}
s \\
2
\end{array}\right)}} .
$$

We use Corollary A.6 to give another sum for $h_{m}^{(n)}(q)$. 
Theorem A.7. If $0 \leq m \leq n-1$,

$$
h_{m}^{(n)}(q)=\left(-q^{2 n-2} ; q^{-2}\right)_{m} \sum_{k=0}^{[m / 2]} \frac{\left(q^{2 n-2 m} ; q^{2}\right)_{2 k}}{\left(q^{4 n+4-4 m} ; q^{4}\right)_{k}\left(q^{4} ; q^{4}\right)_{k}} q^{4 k}
$$

Proof. In Corollary A.6 we replace $q$ by $q^{-2}$, and let

$$
B=q^{-2 n}, \quad R=q^{-4 n+4 m-4}, \quad D^{2}=q^{-2 n+2 m} .
$$

The final expression in Corollary A.6 is

$$
\frac{\left(q^{-2 n+2 m} ; q^{-2}\right)_{m}}{\left(q^{-2} ; q^{-2}\right)_{m}} \frac{\left(q^{-4 n+4 m-2} ; q^{-4}\right)_{m}}{\left(q^{-4 n+4 m-2} ; q^{-2}\right)_{m}} \frac{h_{m}^{(n)}(q)}{C_{m}},
$$

where $h_{m}^{(n)}(q)$ and $C_{m}$ are as in Proposition A.4. The left side of Corollary A.6 is

$$
(-1)^{m} q^{m^{2}-2 n m+m} \sum_{k=0}^{[m / 2]} \frac{\left(q^{2 n-2 m} ; q^{2}\right)_{2 k}}{\left(q^{4 n+4-4 m} ; q^{4}\right)_{k}\left(q^{4} ; q^{4}\right)_{k}} q^{4 k} \text {. }
$$

One verifies that

$$
(-1)^{m} C_{m} \frac{q^{m^{2}-2 n m+m}\left(q^{-2} ; q^{-2}\right)_{m}}{\left(q^{-2 n+2 m} ; q^{-2}\right)_{m}} \frac{\left(q^{-4 n+4 m-2} ; q^{-2}\right)_{m}}{\left(q^{-4 n+4 m-2} ; q^{-4}\right)_{m}}=\left(-q^{2 n-2} ; q^{-2}\right)_{m} .
$$

Proof of Theorem A.1. We rewrite the $k^{\text {th }}$ summand in Theorem A.7

$$
\begin{aligned}
& \left(-q^{2 n-2} ; q^{-2}\right)_{m} \frac{\left(q^{2 n-2 m} ; q^{2}\right)_{2 k}}{\left(q^{4 n+4-4 m} ; q^{4}\right)_{k}\left(q^{4} ; q^{4}\right)_{k}} q^{4 k} \\
& =\left(-q^{2 n-2} ; q^{-2}\right)_{m-2 k} \frac{\left(-q^{2 n-2 m} ; q^{2}\right)_{2 k}\left(q^{2 n-2 m} ; q^{2}\right)_{2 k}}{\left(q^{4 n+4-4 m} ; q^{4}\right)_{k}\left(q^{4} ; q^{4}\right)_{k}} q^{4 k} \\
& =\left(-q^{2 n-2} ; q^{-2}\right)_{m-2 k} \frac{\left(q^{4 n-4 m} ; q^{4}\right)_{2 k}}{\left(q^{4 n+4-4 m} ; q^{4}\right)_{k}\left(q^{4} ; q^{4}\right)_{k}} q^{4 k} \\
& =\left(-q^{2 n-2} ; q^{-2}\right)_{m-2 k}\left[\begin{array}{c}
n-m+2 k \\
k
\end{array}\right]_{q^{4}} \frac{1-q^{4(n-m)}}{1-q^{4(n-m+2 k)}} q^{4 k} \\
& =\left(-q^{2 n-2} ; q^{-2}\right)_{m-2 k}\left(\left[\begin{array}{c}
n-m+2 k-1 \\
k
\end{array}\right]_{q^{4}}-\left[\begin{array}{c}
n-m+2 k-1 \\
k-1
\end{array}\right]_{q^{4}}\right) \text {. }
\end{aligned}
$$

\section{REFERENCES}

[A] A'Campo, Norbert. Tresses, monodromie et le groupe symplectique, Comment. Math. Helv. 54 (1979), no. 2, 318-327.

[An1] Andrews, G. On the difference of successive Gaussian polynomials, J. Stat. Plan. Inf. 34 (1993), no. 1, 19-22. 
[An2] Andrews, G., The Theory of Partitions. Encyclopedia of Mathematics and its Applications, Vol. 2. Addison-Wesley Publishing Co., Reading, Mass.-London-Amsterdam, 1976.

[B] W. Bailey, Products of generalized hypergeometric series, Proc. London Math. Soc. (1928), 242-254.

[CVX1] Chen, T.H., Vilonen, K., Xue, T. Springer correspondence for symmetric spaces. Arxiv.1510.05986.

[CVX2] Chen, T.H., Vilonen, K., Xue, T. Hessenberg varieties, intersections of quadrics, and the Springer correspondence. ArXiv:1511.00617.

[DPS] F. De Mari, C. Procesi, and M. Shayman, Hessenberg varieties. Trans. Amer. Math. Soc. 332 (1992), $529 ? 534$.

[DR] Desale, U. V.; Ramanan, S. Classification of vector bundles of rank 2 on hyperelliptic curves. Invent. Math. 38 (1976/77), no. 2, 161-185.

[Do] Donagi, Ron. Group law on the intersection of two quadrics. Ann. Scuola Norm. Sup. Pisa Cl. Sci. (4) 7 (1980), no. 2, 217-239.

[GKM] M. Goresky, R. Kottwitz, R. Macpherson. Purity of equivalued affine Springer fibers. Representation Theory, 130-146 (2006).

[GR] Gasper, G. and Rahman, M. Basic hypergeometric series. With a foreword by Richard Askey. Encyclopedia of Mathematics and its Applications, Vol. 35. Cambridge University Press, Cambridge, 1990.

[GS] Gessel, I. and Stanton, D., Applications of q-Lagrange inversion to basic hypergeometric series, Trans. Amer. Math. Soc. 277 (1983), no. 1, 173-201.

[KS] Katz, Nicholas M.; Sarnak, Peter. Random matrices, Frobenius eigenvalues, and monodromy. American Mathematical Society Colloquium Publications, 45. American Mathematical Society, Providence, RI, 1999.

[N] Nelson, Graham. The homology of moduli spaces on a Riemann surface as a representation of the mapping class group. Proc. London Math. Soc. (3) 79 (1999), no. 2, 260-282.

[OY] Oblomkov, A and Yun, Z. Geometric representations of graded and rational Cherednik algebras. Advances in Math. 292 (2016), 601-706.

[Ra] Ramanan, S. Orthogonal and spin bundles over hyperelliptic curves. Proc. Indian Acad. Sci. Math. Sci. 90 (1981), no. 2, 151-166.

[R] Reeder, Mark. Desingularizations of some unstable orbit closures. Pacific J. Math. 167 (1995), no. $2,327-343$.

[Re] Reid, Miles. The complete intersection of two or more quadrics. PhD Thesis. Trinity College, Cambridge. 1972.

[S] Sekiguchi, Jirō. The nilpotent subvariety of the vector space associated to a symmetric pair. Publ. Res. Inst. Math. Sci. 20 (1984), no. 1, 155-212.

[St] Stanley, R. Enumerative Combinatorics. Vol. 2. With a foreword by Gian-Carlo Rota and appendix 1 by Sergey Fomin. Cambridge Studies in Advanced Mathematics, 62. Cambridge University Press, Cambridge, 1999.

[T] Tsai, C.C. A formula for certain Shalika germs of ramified unitary groups. ArXiv:1506.08335.

[W] Wang, Xiaoheng. Maximal linear spaces contained in the base loci of pencils of quadrics. ArXiv:1302.2385.

[Z] Zagier, Don. On the cohomology of moduli spaces of rank two vector bundles over curves. The moduli space of curves (Texel Island, 1994), 533-563, Progr. Math., 129, Birkhuser Boston, Boston, MA, 1995. 
Department of Mathematics, Northwestern University, Evanston, 60208, USA

E-mail address: chenth@math.northwestern.edu

Department of Mathematics, Northwestern University, Evanston, 60208, USA, and Department of Mathematics and Statistics, University of Helsinki, Helsinki, 00014, Finland

E-mail address: vilonen@northwestern.edu

School of Mathematics and Statistics, University of Melbourne, ViC 3010, Australia, and Department of Mathematics and Statistics, University of Helsinki, Helsinki, 00014, FINLAND

E-mail address: ting.xue@unimelb.edu.au 